\title{
Influence of Electron-attractor Substituents on the
}

\section{Magnetic Properties of Ni(II) String Complexes.}

\author{
Xavier López, Marc Bénard and Marie-Madeleine Rohmer
}

\author{
Contribution from the Laboratoire de Chimie Quantique, Institut de Chimie, LC3-UMR 7177, \\ CNRS-ULP, Strasbourg, France.
}

Supporting Information 


\section{Complete reference to the Gaussian Software:}

Frisch, M. J.; Trucks, G. W.; Schlegel, H. B.; Scuseria, G. E.; Robb, M. A.; Cheeseman, J. R.; Montgomery, Jr., J. A.; Vreven, T.; Kudin, K. N.; Burant, J. C.; Millam, J. M.; Iyengar, S. S.; Tomasi, J.; Barone, V.; Mennucci, B.; Cossi, M.; Scalmani, G.; Rega, N.; Petersson, G. A.; Nakatsuji, H.; Hada, M.; Ehara, M.; Toyota, K.; Fukuda, R.; Hasegawa, J.; Ishida, M.; Nakajima, T.; Honda, Y.; Kitao, O.; Nakai, H.; Klene, M.; Li, X.; Knox, J. E.; Hratchian, H. P.; Cross, J. B.; Adamo, C.; Jaramillo, J.; Gomperts, R.; Stratmann, R. E.; Yazyev, O.; Austin, A. J.; Cammi, R.; Pomelli, C.; Ochterski, J. W.; Ayala, P. Y.; Morokuma, K.; Voth, G. A.; Salvador, P.; Dannenberg, J. J.; Zakrzewski, V. G.; Dapprich, S.; Daniels, A. D.; Strain, M. C.; Farkas, O.; Malick, D. K.; Rabuck, A. D.; Raghavachari, K.; Foresman, J. B.; Ortiz, J. V.; Cui, Q.; Baboul, A. G.; Clifford, S.; Cioslowski, J.; Stefanov, B. B.; Liu, G.; Liashenko, A.; Piskorz, P.; Komaromi, I.; Martin, R. L.; Fox, D. J.; Keith, T.; Al-Laham, M. A.; Peng, C. Y.; Nanayakkara, A.; Challacombe, M.; Gill, P. M. W.; Johnson, B.; Chen, W.;

Wong, M. W.; Gonzalez, C.; Pople, J. A. Gaussian 03, Revision B.05; Gaussian, Inc., Pittsburgh PA, 2003. 
Total Energies and Cartesian Coordinates and Population Analysis obtained from DFT/B3LYP and DFT/UB3LYP calculations Basis sets: C, O, H: D95V; Ni: Lanl2DZ; S: D95(2d); N: D95(d)

$\mathrm{Ni}_{3}(\mathrm{BAP})_{4}\left(\mathrm{D}_{4}\right.$ symmetry $)$
Diamagnetic Singlet
55 atoms
$\mathrm{E}=-1939.26170$ Hartrees

\begin{tabular}{ccccc} 
& \multicolumn{3}{c}{ Cartesian Coordinates } & Mulliken \\
& $\mathrm{y}$ & $\mathrm{y}$ & $\mathrm{z}$ & Atomic \\
& & & & \\
& & & & \\
$\mathrm{Ni}$ & & & & \\
$\mathrm{Ni}$ & 0.000000 & 0.000000 & 2.426202 & 0.043 \\
$\mathrm{Ni}$ & 0.000000 & 0.000000 & 0.000000 & 0.155 \\
$\mathrm{Ni}$ & 0.000000 & 0.000000 & -2.426202 & 0.043 \\
$\mathrm{~N}$ & -0.367416 & 1.888903 & 2.310726 & -0.628 \\
$\mathrm{~N}$ & 0.000000 & 1.951310 & 0.000000 & -0.361 \\
$\mathrm{~N}$ & 0.367416 & 1.888403 & -2.310726 & -0.628 \\
$\mathrm{C}$ & 0.170625 & 2.619814 & -1.194808 & 0.549 \\
$\mathrm{C}$ & 0.164572 & 4.062266 & -1.208300 & -0.442 \\
$\mathrm{C}$ & 0.000000 & 4.751050 & 0.000000 & -0.138 \\
$\mathrm{C}$ & -0.164572 & 4.062266 & 1.208300 & -0.442 \\
$\mathrm{C}$ & -0.170625 & 2.619814 & 1.194808 & 0.549 \\
$\mathrm{H}$ & 0.292863 & 4.580750 & -2.156560 & 0.165 \\
$\mathrm{H}$ & 0.000000 & 5.844409 & 0.000000 & 0.161 \\
$\mathrm{H}$ & -0.292863 & 4.580750 & 2.156560 & 0.165 \\
$\mathrm{H}$ & 0.398402 & 2.476973 & -3.140706 & 0.244 \\
$\mathrm{H}$ & -0.398402 & 2.476973 & 3.140706 & 0.244 \\
$\mathrm{~N}$ & -1.888903 & -0.367416 & 2.310726 & -0.628 \\
$\mathrm{~N}$ & -1.951310 & 0.000000 & 0.000000 & -0.361 \\
$\mathrm{~N}$ & -1.888903 & 0.367416 & -2.310726 & -0.628 \\
$\mathrm{C}$ & -2.619814 & 0.170625 & -1.194808 & 0.549 \\
$\mathrm{C}$ & -4.062266 & 0.164572 & -1.208300 & -0.442 \\
$\mathrm{C}$ & -4.751050 & 0.000000 & 0.000000 & -0.138 \\
$\mathrm{C}$ & -4.062266 & -0.164572 & 1.208300 & -0.442 \\
$\mathrm{C}$ & -2.619814 & -0.170625 & 1.194808 & 0.549 \\
$\mathrm{H}$ & -4.580750 & 0.292863 & -2.156560 & 0.165 \\
$\mathrm{H}$ & -5.844409 & 0.000000 & 0.000000 & 0.161 \\
$\mathrm{H}$ & -4.580750 & -0.292863 & 2.156560 & 0.165 \\
$\mathrm{H}$ & -2.476973 & 0.398402 & -3.140706 & 0.244 \\
$\mathrm{H}$ & -2.476973 & -0.398402 & 3.140706 & 0.244 \\
$\mathrm{~N}$ & 0.367416 & -1.888903 & 2.310726 & -0.628 \\
$\mathrm{~N}$ & 0.000000 & -1.951310 & 0.000000 & -0.361 \\
$\mathrm{~N}$ & -0.367416 & -1.888403 & -2.310726 & -0.628 \\
$\mathrm{C}$ & -0.170625 & -2.619814 & -1.194808 & 0.549 \\
$\mathrm{C}$ & -0.164572 & -4.062266 & -1.208300 & -0.442 \\
$\mathrm{C}$ & 0.000000 & -4.751050 & 0.000000 & -0.138 \\
$\mathrm{C}$ & 0.164572 & -4.062266 & 1.208300 & -0.442 \\
$\mathrm{C}$ & 0.170625 & -2.619814 & 1.194808 & 0.549
\end{tabular}




$\begin{array}{lrrrr}\mathrm{H} & -0.292863 & -4.580750 & -2.156560 & 0.165 \\ \mathrm{H} & 0.000000 & -5.844409 & 0.000000 & 0.161 \\ \mathrm{H} & 0.292863 & -4.580750 & 2.156560 & 0.165 \\ \mathrm{H} & -0.398402 & -2.476973 & -3.140706 & 0.244 \\ \mathrm{H} & 0.398402 & -2.476973 & 3.140706 & 0.244 \\ \mathrm{~N} & 1.888903 & 0.367416 & 2.310726 & -0.628 \\ \mathrm{~N} & 1.951310 & 0.000000 & 0.000000 & -0.361 \\ \mathrm{~N} & 1.888903 & -0.367416 & -2.310726 & -0.628 \\ \mathrm{C} & 2.619814 & -0.170625 & -1.194808 & 0.549 \\ \mathrm{C} & 4.062266 & -0.164572 & -1.208300 & -0.442 \\ \mathrm{C} & 4.751050 & 0.000000 & 0.000000 & -0.138 \\ \mathrm{C} & 4.062266 & 0.164572 & 1.208300 & -0.442 \\ \mathrm{C} & 2.619814 & 0.170625 & 1.194808 & 0.549 \\ \mathrm{H} & 4.580750 & -0.292863 & -2.156560 & 0.165 \\ \mathrm{H} & 5.844409 & 0.000000 & 0.000000 & 0.161 \\ \mathrm{H} & 4.580750 & 0.292863 & 2.156560 & 0.165 \\ \mathrm{H} & 2.476973 & -0.398402 & -3.140706 & 0.244 \\ \mathrm{H} & 2.476973 & 0.398402 & 3.140706 & 0.244\end{array}$

$\mathrm{Ni}_{3}(\mathrm{BAP})_{4}\left(\mathrm{D}_{4}\right.$ symmetry $)$

Triplet

55 atoms

$\mathrm{E}=-1939.25410$ Hartrees

$\mathrm{S}^{2}=2.0121$

$\begin{array}{rrrrrr}\mathrm{Ni} & 0.000000 & 0.000000 & 2.380689 & 0.059 & 0.092 \\ \mathrm{Ni} & 0.000000 & 0.000000 & 0.000000 & 0.268 & 1.504 \\ \mathrm{Ni} & 0.000000 & 0.000000 & -2.380689 & 0.059 & 0.092 \\ \mathrm{~N} & -0.097245 & 1.935880 & 2.324702 & -0.635 & -0.002 \\ \mathrm{~N} & 0.000000 & 2.045001 & 0.000000 & -0.366 & 0.083 \\ \mathrm{~N} & 0.097245 & 1.935880 & -2.324702 & -0.635 & -0.002 \\ \mathrm{C} & 0.008514 & 2.696472 & -1.201613 & 0.528 & -0.003 \\ \mathrm{C} & -0.023901 & 4.137435 & -1.225121 & -0.441 & 0.004 \\ \mathrm{C} & 0.000000 & 4.822355 & 0.000000 & -0.136 & -0.004 \\ \mathrm{C} & 0.023901 & 4.137435 & 1.225121 & -0.441 & 0.004 \\ \mathrm{C} & -0.008514 & 2.696472 & 1.201613 & 0.528 & -0.003 \\ \mathrm{H} & -0.019326 & 4.662723 & -2.178361 & 0.171 & 0.001 \\ \mathrm{H} & 0.000000 & 5.915912 & 0.000000 & 0.162 & 0.000 \\ \mathrm{H} & 0.019326 & 4.662723 & 2.178361 & 0.171 & 0.001 \\ \mathrm{H} & -0.190259 & 2.458013 & -3.150617 & 0.251 & 0.000 \\ \mathrm{H} & 0.190259 & 2.458013 & 3.150617 & 0.251 & 0.000 \\ \mathrm{~N} & -1.935880 & -0.097245 & 2.324702 & -0.635 & -0.002 \\ \mathrm{~N} & -2.045001 & 0.000000 & 0.000000 & -0.366 & 0.083 \\ \mathrm{~N} & -1.935880 & 0.097245 & -2.324702 & -0.635 & -0.002 \\ \mathrm{C} & -2.696472 & 0.008514 & -1.201613 & 0.528 & -0.003 \\ \mathrm{C} & -4.137435 & -0.023901 & -1.225121 & -0.441 & 0.004 \\ \mathrm{C} & -4.822355 & 0.000000 & 0.000000 & -0.136 & -0.004 \\ \mathrm{C} & -4.137435 & 0.023901 & 1.225121 & -0.441 & 0.004\end{array}$




$\begin{array}{rrrrrr}\mathrm{C} & -2.696472 & -0.008514 & 1.201613 & 0.528 & -0.003 \\ \mathrm{H} & -4.662723 & -0.019326 & -2.178361 & 0.171 & 0.001 \\ \mathrm{H} & -5.915912 & 0.000000 & 0.000000 & 0.161 & 0.000 \\ \mathrm{H} & -4.662723 & 0.019326 & 2.178361 & 0.171 & 0.001 \\ \mathrm{H} & -2.458013 & -0.190259 & -3.150617 & 0.251 & 0.000 \\ \mathrm{H} & -2.458013 & 0.190259 & 3.150617 & 0.251 & 0.000 \\ \mathrm{~N} & 0.097245 & -1.935880 & 2.324702 & -0.635 & -0.002 \\ \mathrm{~N} & 0.000000 & -2.045001 & 0.000000 & -0.366 & 0.083 \\ \mathrm{~N} & -0.097245 & -1.935880 & -2.324702 & -0.635 & -0.002 \\ \mathrm{C} & -0.008514 & -2.696472 & -1.201613 & 0.528 & -0.003 \\ \mathrm{C} & 0.023901 & -4.137435 & -1.225121 & -0.441 & 0.004 \\ \mathrm{C} & 0.000000 & -4.822355 & 0.000000 & -0.136 & -0.004 \\ \mathrm{C} & -0.023901 & -4.137435 & 1.225121 & -0.441 & 0.004 \\ \mathrm{C} & 0.008514 & -2.696472 & 1.201613 & 0.528 & -0.003 \\ \mathrm{H} & 0.019326 & -4.662723 & -2.178361 & 0.171 & 0.001 \\ \mathrm{H} & 0.000000 & -5.915912 & 0.000000 & 0.162 & 0.000 \\ \mathrm{H} & -0.019326 & -4.662723 & 2.178361 & 0.171 & 0.001 \\ \mathrm{H} & 0.190259 & -2.458013 & -3.150617 & 0.251 & 0.000 \\ \mathrm{H} & -0.190259 & -2.458013 & 3.150617 & 0.251 & 0.000 \\ \mathrm{~N} & 1.935880 & 0.097245 & 2.324702 & -0.635 & -0.002 \\ \mathrm{~N} & 2.045001 & 0.000000 & 0.000000 & -0.366 & 0.083 \\ \mathrm{~N} & 1.935880 & -0.097245 & -2.324702 & -0.635 & -0.002 \\ \mathrm{C} & 2.696472 & -0.008514 & -1.201613 & 0.528 & -0.003 \\ \mathrm{C} & 4.137435 & 0.023901 & -1.225121 & -0.441 & 0.004 \\ \mathrm{C} & 4.822355 & 0.000000 & 0.000000 & -0.136 & -0.004 \\ \mathrm{C} & 4.137435 & -0.023901 & 1.225121 & -0.441 & 0.004 \\ \mathrm{C} & 2.696472 & 0.008514 & 1.201613 & 0.528 & -0.003 \\ \mathrm{H} & 4.662723 & 0.019326 & -2.178361 & 0.171 & 0.001 \\ \mathrm{H} & 5.915912 & 0.000000 & 0.000000 & 0.161 & 0.000 \\ \mathrm{H} & 4.662723 & -0.019326 & 2.178361 & 0.171 & 0.001 \\ \mathrm{H} & 2.458013 & 0.190259 & -3.150617 & 0.251 & 0.000 \\ \mathrm{H} & 2.458013 & -0.190259 & 3.150617 & 0.251 & 0.000\end{array}$

$\mathrm{Ni}_{3}(\mathrm{BAP})_{4}\left(\mathrm{D}_{4}\right.$ symmetry)

Quintet

55 atoms

$\mathrm{E}=-1939.19361$ Hartrees

$S^{2}=6.0801$

$\begin{array}{rrrrrr}\mathrm{Ni} & 0.000000 & 0.000000 & 2.405068 & 0.065 & 1.242 \\ \mathrm{Ni} & 0.000000 & 0.000000 & 0.000000 & 0.175 & 0.321 \\ \mathrm{Ni} & 0.000000 & 0.000000 & -2.405068 & 0.065 & 1.242 \\ \mathrm{~N} & -0.631421 & 1.944016 & 2.252613 & -0.629 & 0.116 \\ \mathrm{~N} & 0.000000 & 1.980446 & 0.000000 & -0.366 & -0.012 \\ \mathrm{~N} & 0.631421 & 1.944016 & -2.252613 & -0.629 & 0.116 \\ \mathrm{C} & 0.337774 & 2.658875 & -1.159987 & 0.533 & -0.024 \\ \mathrm{C} & 0.359748 & 4.108520 & -1.158393 & -0.417 & 0.087 \\ \mathrm{C} & 0.000000 & 4.804935 & 0.000000 & -0.146 & -0.038 \\ \mathrm{C} & -0.359748 & 4.108520 & 1.158393 & -0.417 & 0.087 \\ \mathrm{C} & -0.337774 & 2.658875 & 1.159987 & 0.533 & -0.024\end{array}$




\begin{tabular}{|c|c|c|c|c|c|}
\hline $\mathrm{H}$ & 0.634116 & 4.620583 & -2.078844 & 0.166 & -0.006 \\
\hline $\mathrm{H}$ & 0.000000 & 5.897975 & 0.000000 & 0.163 & 0.003 \\
\hline $\mathrm{H}$ & -0.634116 & 4.620583 & 2.078844 & 0.166 & -0.006 \\
\hline $\mathrm{H}$ & 0.884274 & 2.564315 & 3.022128 & 0.232 & 0.000 \\
\hline $\mathrm{H}$ & -0.884274 & 2.564315 & -3.022128 & 0.232 & 0.000 \\
\hline $\mathrm{N}$ & -1.944016 & -0.631421 & 2.252613 & -0.629 & 0.116 \\
\hline $\mathrm{N}$ & -1.980446 & 0.000000 & 0.000000 & -0.366 & -0.012 \\
\hline $\mathrm{N}$ & -1.944016 & 0.631421 & -2.252613 & -0.629 & 0.116 \\
\hline $\mathrm{C}$ & -2.658875 & 0.337774 & -1.159987 & 0.533 & -0.024 \\
\hline $\mathrm{C}$ & -4.108520 & 0.359748 & -1.158393 & -0.417 & 0.087 \\
\hline $\mathrm{C}$ & -4.804935 & 0.000000 & 0.000000 & -0.146 & -0.038 \\
\hline $\mathrm{C}$ & -4.108520 & -0.359748 & 1.158393 & -0.417 & 0.087 \\
\hline $\mathrm{C}$ & -2.658875 & -0.337774 & 1.159987 & 0.533 & -0.024 \\
\hline $\mathrm{H}$ & -4.620583 & 0.634116 & -2.078844 & 0.166 & -0.006 \\
\hline $\mathrm{H}$ & -5.897975 & 0.000000 & 0.000000 & 0.163 & 0.003 \\
\hline $\mathrm{H}$ & -4.620583 & -0.634116 & 2.078844 & 0.166 & -0.006 \\
\hline $\mathrm{H}$ & -2.564315 & 0.884274 & -3.022128 & 0.232 & 0.000 \\
\hline $\mathrm{H}$ & -2.564315 & -0.884274 & 3.022128 & 0.232 & 0.000 \\
\hline $\mathrm{N}$ & 0.631421 & -1.944016 & 2.252613 & -0.629 & 0.116 \\
\hline $\mathrm{N}$ & 0.000000 & -1.980446 & 0.000000 & -0.366 & -0.012 \\
\hline $\mathrm{N}$ & -0.631421 & -1.944016 & -2.252613 & -0.629 & 0.116 \\
\hline $\mathrm{C}$ & -0.337774 & -2.658875 & -1.159987 & 0.533 & -0.024 \\
\hline $\mathrm{C}$ & -0.359748 & -4.108520 & -1.158393 & -0.417 & 0.087 \\
\hline $\mathrm{C}$ & 0.000000 & -4.804935 & 0.000000 & -0.146 & -0.038 \\
\hline $\mathrm{C}$ & 0.359748 & -4.108520 & 1.158393 & -0.417 & 0.087 \\
\hline $\mathrm{C}$ & 0.337774 & -2.658875 & 1.159987 & 0.533 & -0.024 \\
\hline $\mathrm{H}$ & -0.634116 & -4.620583 & -2.078844 & 0.166 & -0.006 \\
\hline $\mathrm{H}$ & 0.000000 & -5.897975 & 0.000000 & 0.163 & 0.003 \\
\hline $\mathrm{H}$ & 0.634116 & -4.620583 & 2.078844 & 0.166 & -0.006 \\
\hline $\mathrm{H}$ & -0.884274 & -2.564315 & 3.022128 & 0.232 & 0.000 \\
\hline $\mathrm{H}$ & 0.884274 & -2.564315 & -3.022128 & 0.232 & 0.000 \\
\hline $\mathrm{N}$ & 1.944016 & 0.631421 & 2.252613 & -0.629 & 0.116 \\
\hline $\mathrm{N}$ & 1.980446 & 0.000000 & 0.000000 & -0.366 & -0.012 \\
\hline $\mathrm{N}$ & 1.944016 & -0.631421 & -2.252613 & -0.629 & 0.116 \\
\hline $\mathrm{C}$ & 2.658875 & -0.337774 & -1.159987 & 0.533 & -0.024 \\
\hline $\mathrm{C}$ & 4.108520 & -0.359748 & -1.158393 & -0.417 & 0.087 \\
\hline $\mathrm{C}$ & 4.804935 & 0.000000 & 0.000000 & -0.146 & -0.038 \\
\hline $\mathrm{C}$ & 4.108520 & 0.359748 & 1.158393 & -0.417 & 0.087 \\
\hline $\mathrm{C}$ & 2.658875 & 0.337774 & 1.159987 & 0.533 & -0.024 \\
\hline $\mathrm{H}$ & 4.620583 & -0.634116 & -2.078844 & 0.166 & -0.006 \\
\hline $\mathrm{H}$ & 5.897975 & 0.000000 & 0.000000 & 0.163 & 0.003 \\
\hline $\mathrm{H}$ & 4.620583 & 0.634116 & 2.078844 & 0.166 & -0.006 \\
\hline $\mathrm{H}$ & 2.564315 & -0.884274 & -3.022128 & 0.232 & 0.000 \\
\hline $\mathrm{H}$ & 2.564315 & 0.884274 & 3.022128 & 0.232 & 0.000 \\
\hline
\end{tabular}


$\mathrm{Ni}_{3}\left[\left(\mathrm{HSO}_{2}\right)_{2}-\mathrm{BAP}\right)_{4}\left(\mathrm{D}_{4}\right.$ symmetry $)$

Diamagnetic Singlet

79 atoms

$E=-3223.505827$ Hartrees

$\begin{array}{rrrrr}\mathrm{Ni} & 0.000000 & 0.000000 & 2.393322 & -0.073 \\ \mathrm{Ni} & 0.000000 & 0.000000 & 0.000000 & 0.091 \\ \mathrm{Ni} & 0.000000 & 0.000000 & -2.393322 & -0.073 \\ \mathrm{~N} & -0.810116 & 1.795359 & 2.202521 & -0.549 \\ \mathrm{~N} & 0.000000 & 1.935005 & 0.000000 & -0.352 \\ \mathrm{~N} & 0.810116 & 1.795359 & -2.202521 & -0.549 \\ \mathrm{C} & 0.421249 & 2.601968 & -1.122344 & 0.579 \\ \mathrm{C} & 0.426267 & 4.017313 & -1.138471 & -0.328 \\ \mathrm{C} & 0.000000 & 4.704401 & 0.000000 & -0.163 \\ \mathrm{C} & -0.426267 & 4.017313 & 1.138471 & -0.328 \\ \mathrm{C} & -0.421249 & 2.601968 & 1.122344 & 0.579 \\ \mathrm{H} & 0.778052 & 4.553711 & -2.006572 & 0.248 \\ \mathrm{H} & 0.000000 & 5.792331 & 0.000000 & 0.209 \\ \mathrm{H} & -0.778052 & 4.553711 & 2.006572 & 0.248 \\ \mathrm{~N} & -1.795359 & -0.810116 & 2.202521 & -0.549 \\ \mathrm{~N} & -1.935005 & 0.000000 & 0.000000 & -0.352 \\ \mathrm{~N} & -1.795359 & 0.810116 & -2.202521 & -0.549 \\ \mathrm{C} & -2.601968 & 0.421249 & -1.122344 & 0.579 \\ \mathrm{C} & -4.017313 & 0.426267 & -1.138471 & -0.328 \\ \mathrm{C} & -4.704401 & 0.000000 & 0.000000 & -0.163 \\ \mathrm{C} & -4.017313 & -0.426267 & 1.138471 & -0.328 \\ \mathrm{C} & -2.601968 & -0.421249 & 1.122344 & 0.579 \\ \mathrm{H} & -4.553711 & 0.778052 & -2.006572 & 0.248 \\ \mathrm{H} & -5.792331 & 0.000000 & 0.000000 & 0.209 \\ \mathrm{H} & -4.553711 & -0.778052 & 2.006572 & 0.248 \\ \mathrm{~N} & 0.810116 & -1.795359 & 2.202521 & -0.549 \\ \mathrm{~N} & 0.000000 & -1.935005 & 0.000000 & -0.352 \\ \mathrm{~N} & -0.810116 & -1.795359 & -2.202521 & -0.549 \\ \mathrm{C} & -0.421249 & -2.601968 & -1.122344 & 0.579 \\ \mathrm{C} & -0.426267 & -4.017313 & -1.138471 & -0.328 \\ \mathrm{C} & 0.000000 & -4.704401 & 0.000000 & -0.163 \\ \mathrm{C} & 0.426267 & -4.017313 & 1.138471 & -0.328 \\ \mathrm{C} & 0.421249 & -2.601968 & 1.122344 & 0.579 \\ \mathrm{C} & 2.601968 & 0.421249 & 1.122344 & 0.579 \\ \mathrm{H} & -0.778052 & -4.553711 & -2.006572 & 0.248 \\ \mathrm{H} & 0.000000 & -5.792331 & 0.000000 & 0.209 \\ \mathrm{H} & 0.778052 & -4.553711 & 2.006572 & 0.248 \\ \mathrm{~N} & 1.795359 & 0.810116 & 2.202521 & -0.549 \\ \mathrm{~N} & 1.935005 & 0.000000 & 0.000000 & -0.352 \\ \mathrm{~N} & 1.795359 & -0.810116 & -2.202521 & -0.549 \\ \mathrm{C} & 2.601968 & -0.421249 & -1.122344 & 0.579 \\ \mathrm{C} & 4.017313 & -0.426267 & -1.138471 & -0.328 \\ \mathrm{H} & 4.017313 & 0.000000 & 0.000000 & -0.163 \\ \mathrm{H} & & & & -0.328 \\ \mathrm{H} & & & \end{array}$




$\begin{array}{lrrrr}\mathrm{H} & 4.553711 & -0.778052 & -2.006572 & 0.248 \\ \mathrm{H} & 5.792331 & 0.000000 & 0.000000 & 0.209 \\ \mathrm{H} & 4.553711 & 0.778052 & 2.006572 & 0.248 \\ \mathrm{~S} & 1.536647 & 2.452487 & -3.500069 & 0.466 \\ \mathrm{~S} & -1.536647 & -2.452487 & -3.500069 & 0.466 \\ \mathrm{~S} & -1.536647 & 2.452487 & 3.500069 & 0.466 \\ \mathrm{~S} & 1.536647 & -2.452487 & 3.500069 & 0.466 \\ \mathrm{H} & 0.530572 & 3.073380 & -4.170740 & 0.297 \\ \mathrm{H} & -0.530572 & -3.073380 & -4.170740 & 0.297 \\ \mathrm{H} & 0.530572 & -3.073380 & 4.170740 & 0.297 \\ \mathrm{H} & -0.530572 & 3.073380 & 4.170740 & 0.297 \\ \mathrm{O} & 1.973003 & 1.352447 & -4.371484 & -0.391 \\ \mathrm{O} & -1.973003 & -1.352447 & -4.371484 & -0.391 \\ \mathrm{O} & 1.973003 & -1.352447 & 4.371484 & -0.391 \\ \mathrm{O} & -1.973003 & 1.352447 & 4.371484 & -0.391 \\ \mathrm{O} & 2.512064 & 3.496316 & -3.148426 & -0.411 \\ \mathrm{O} & -2.512064 & -3.496316 & -3.148426 & -0.411 \\ \mathrm{O} & -2.512064 & 3.496316 & 3.148426 & -0.411 \\ \mathrm{O} & 2.512064 & -3.496316 & 3.148426 & -0.411 \\ \mathrm{~S} & 2.452487 & -1.536647 & -3.500069 & 0.466 \\ \mathrm{~S} & -2.452487 & 1.536647 & -3.500069 & 0.466 \\ \mathrm{~S} & 2.452487 & 1.536647 & 3.500069 & 0.466 \\ \mathrm{~S} & -2.452487 & -1.536647 & 3.500069 & 0.466 \\ \mathrm{H} & 3.073380 & -0.530572 & -4.170740 & 0.297 \\ \mathrm{H} & -3.073380 & 0.530572 & -4.170740 & 0.297 \\ \mathrm{H} & -3.073380 & -0.530572 & 4.170740 & 0.297 \\ \mathrm{H} & 3.073380 & 0.530572 & 4.170740 & 0.297 \\ \mathrm{O} & 1.352447 & -1.973003 & -4.371484 & -0.391 \\ \mathrm{O} & -1.352447 & 1.973003 & -4.371484 & -0.391 \\ \mathrm{O} & -1.352447 & -1.973003 & 4.371484 & -0.391 \\ \mathrm{O} & 1.352447 & 1.973003 & 4.371484 & -0.391 \\ \mathrm{O} & 3.496316 & -2.512064 & -3.148426 & -0.411 \\ \mathrm{O} & -3.496316 & 2.512064 & -3.148426 & -0.411 \\ \mathrm{O} & 3.496316 & 2.512064 & 3.148426 & -0.411 \\ \mathrm{O} & -3.496316 & -2.512064 & 3.148426 & -0.411 \\ & & & & \\ & & & & \\ & & & \end{array}$

$\mathrm{Ni}_{3}\left[\left(\mathrm{HSO}_{2}\right)_{2}-\mathrm{BAP}\right)_{4}\left(\mathrm{D}_{4}\right.$ symmetry $)$

Triplet

79 atoms

$\mathrm{E}=-3223.455174$ Hartrees

$\mathrm{S}^{2}=2.0083$

$\begin{array}{rrrrrr}\mathrm{Ni} & 0.000000 & 0.000000 & 2.391688 & -0.097 & -0.004 \\ \mathrm{Ni} & 0.000000 & 0.000000 & 0.000000 & 0.187 & 1.536 \\ \mathrm{Ni} & 0.000000 & 0.000000 & -2.391688 & -0.097 & -0.004 \\ \mathrm{~N} & -0.748971 & 1.845191 & 2.215526 & -0.539 & 0.004 \\ \mathrm{~N} & 0.000000 & 2.013063 & 0.000000 & -0.382 & 0.107 \\ \mathrm{~N} & 0.748971 & 1.845191 & -2.215526 & -0.539 & 0.004 \\ \mathrm{C} & 0.383848 & 2.669964 & -1.136312 & 0.564 & -0.002\end{array}$




\begin{tabular}{|c|c|c|c|c|c|}
\hline $\mathrm{C}$ & 0.387471 & 4.086007 & -1.157210 & -0.321 & 0.002 \\
\hline $\mathrm{C}$ & 0.000000 & 4.769039 & 0.000000 & -0.162 & 0.003 \\
\hline $\mathrm{C}$ & -0.387471 & 4.086007 & 1.157210 & -0.321 & 0.002 \\
\hline $\mathrm{C}$ & -0.383848 & 2.669964 & 1.136312 & 0.564 & -0.002 \\
\hline $\mathrm{H}$ & 0.710722 & 4.626710 & -2.033852 & 0.248 & 0.001 \\
\hline $\mathrm{H}$ & 0.000000 & 5.857232 & 0.000000 & 0.208 & -0.000 \\
\hline $\mathrm{H}$ & -0.710722 & 4.626710 & 2.033852 & 0.248 & 0.001 \\
\hline $\mathrm{N}$ & -1.845191 & -0.748971 & 2.215526 & -0.539 & 0.004 \\
\hline $\mathrm{N}$ & -2.013063 & 0.000000 & 0.000000 & -0.382 & 0.107 \\
\hline $\mathrm{N}$ & -1.845191 & 0.748971 & -2.215526 & -0.539 & 0.004 \\
\hline $\mathrm{C}$ & -2.669964 & 0.383848 & -1.136312 & 0.564 & -0.002 \\
\hline $\mathrm{C}$ & -4.086007 & 0.387471 & -1.157210 & -0.321 & 0.002 \\
\hline $\mathrm{C}$ & -4.769039 & 0.000000 & 0.000000 & -0.162 & 0.003 \\
\hline $\mathrm{C}$ & -4.086007 & -0.387471 & 1.157210 & -0.321 & 0.002 \\
\hline $\mathrm{C}$ & -2.669964 & -0.383848 & 1.136312 & 0.564 & -0.002 \\
\hline $\mathrm{H}$ & -4.626710 & 0.710722 & -2.033852 & 0.248 & 0.001 \\
\hline $\mathrm{H}$ & -5.857232 & 0.000000 & 0.000000 & 0.208 & -0.000 \\
\hline $\mathrm{H}$ & -4.626710 & -0.710722 & 2.033852 & 0.248 & 0.001 \\
\hline $\mathrm{N}$ & 0.748971 & -1.845191 & 2.215526 & -0.539 & 0.004 \\
\hline $\mathrm{N}$ & 0.000000 & -2.013063 & 0.000000 & -0.382 & 0.107 \\
\hline $\mathrm{N}$ & -0.748971 & -1.845191 & -2.215526 & -0.539 & 0.004 \\
\hline $\mathrm{C}$ & -0.383848 & -2.669964 & -1.136312 & 0.564 & -0.002 \\
\hline $\mathrm{C}$ & -0.387471 & -4.086007 & -1.157210 & -0.321 & 0.002 \\
\hline $\mathrm{C}$ & 0.000000 & -4.769039 & 0.000000 & -0.162 & 0.003 \\
\hline $\mathrm{C}$ & 0.387471 & -4.086007 & 1.157210 & -0.321 & 0.002 \\
\hline $\mathrm{C}$ & 0.383848 & -2.669964 & 1.136312 & 0.564 & -0.002 \\
\hline $\mathrm{H}$ & -0.710722 & -4.626710 & -2.033852 & 0.248 & 0.001 \\
\hline $\mathrm{H}$ & 0.000000 & -5.857232 & 0.000000 & 0.208 & -0.000 \\
\hline $\mathrm{H}$ & 0.710722 & -4.626710 & 2.033852 & 0.248 & 0.001 \\
\hline $\mathrm{N}$ & 1.845191 & 0.748971 & 2.215526 & -0.539 & 0.004 \\
\hline $\mathrm{N}$ & 2.013063 & 0.000000 & 0.000000 & -0.382 & 0.107 \\
\hline $\mathrm{N}$ & 1.845191 & -0.748971 & -2.215526 & -0.539 & 0.004 \\
\hline $\mathrm{C}$ & 2.669964 & -0.383848 & -1.136312 & 0.564 & -0.002 \\
\hline $\mathrm{C}$ & 4.086007 & -0.387471 & -1.157210 & -0.321 & 0.002 \\
\hline $\mathrm{C}$ & 4.769039 & 0.000000 & 0.000000 & -0.162 & 0.003 \\
\hline $\mathrm{C}$ & 4.086007 & 0.387471 & 1.157210 & -0.321 & 0.002 \\
\hline $\mathrm{C}$ & 2.669964 & 0.383848 & 1.136312 & 0.564 & -0.002 \\
\hline $\mathrm{H}$ & 4.626710 & -0.710722 & -2.033852 & 0.248 & 0.001 \\
\hline $\mathrm{H}$ & 5.857232 & 0.000000 & 0.000000 & 0.208 & -0.000 \\
\hline $\mathrm{H}$ & 4.626710 & 0.710722 & 2.033852 & 0.248 & 0.001 \\
\hline $\mathrm{S}$ & 1.444931 & 2.496117 & -3.534401 & 0.470 & -0.000 \\
\hline S & -1.444931 & -2.496117 & -3.534401 & 0.470 & -0.000 \\
\hline$S$ & -1.444931 & 2.496117 & 3.534401 & 0.470 & -0.000 \\
\hline $\mathrm{S}$ & 1.444931 & -2.496117 & 3.534401 & 0.470 & -0.000 \\
\hline $\mathrm{H}$ & 0.419818 & 3.097273 & -4.193642 & 0.294 & -0.000 \\
\hline $\mathrm{H}$ & -0.419818 & -3.097273 & -4.193642 & 0.294 & -0.000 \\
\hline $\mathrm{H}$ & 0.419818 & -3.097273 & 4.193642 & 0.294 & -0.000 \\
\hline $\mathrm{H}$ & -0.419818 & 3.097273 & 4.193642 & 0.294 & -0.000 \\
\hline $\mathrm{O}$ & 1.880542 & 1.392259 & -4.400491 & -0.387 & 0.000 \\
\hline $\mathrm{O}$ & -1.880542 & -1.392259 & -4.400491 & -0.387 & 0.000 \\
\hline
\end{tabular}




$\begin{array}{lrrrrr}\mathrm{O} & 1.880542 & -1.392259 & 4.400491 & -0.387 & 0.000 \\ \mathrm{O} & -1.880542 & 1.392259 & 4.400491 & -0.387 & 0.000 \\ \mathrm{O} & 2.412343 & 3.557381 & -3.213006 & -0.411 & -0.000 \\ \mathrm{O} & -2.412343 & -3.557381 & -3.213006 & -0.411 & -0.000 \\ \mathrm{O} & -2.412343 & 3.557381 & 3.213006 & -0.411 & -0.000 \\ \mathrm{O} & 2.412343 & -3.557381 & 3.213006 & -0.411 & -0.000 \\ \mathrm{~S} & 2.496117 & -1.444931 & -3.534401 & 0.470 & -0.000 \\ \mathrm{~S} & -2.496117 & 1.444931 & -3.534401 & 0.470 & -0.000 \\ \mathrm{~S} & 2.496117 & 1.444931 & 3.534401 & 0.470 & -0.000 \\ \mathrm{~S} & -2.496117 & -1.444931 & 3.534401 & 0.470 & -0.000 \\ \mathrm{H} & 3.097273 & -0.419818 & -4.193642 & 0.294 & -0.000 \\ \mathrm{H} & -3.097273 & 0.419818 & -4.193642 & 0.294 & -0.000 \\ \mathrm{H} & -3.097273 & -0.419818 & 4.193642 & 0.294 & -0.000 \\ \mathrm{H} & 3.097273 & 0.419818 & 4.193642 & 0.294 & -0.000 \\ \mathrm{O} & 1.392259 & -1.880542 & -4.400491 & -0.387 & 0.000 \\ \mathrm{O} & -1.392259 & 1.880542 & -4.400491 & -0.387 & 0.000 \\ \mathrm{O} & -1.392259 & -1.880542 & 4.400491 & -0.387 & 0.000 \\ \mathrm{O} & 1.392259 & 1.880542 & 4.400491 & -0.387 & 0.000 \\ \mathrm{O} & 3.557381 & -2.412343 & -3.213006 & -0.411 & -0.000 \\ \mathrm{O} & -3.557381 & 2.412343 & -3.213006 & -0.411 & -0.000 \\ \mathrm{O} & 3.557381 & 2.412343 & 3.213006 & -0.411 & -0.000 \\ \mathrm{O} & -3.557381 & -2.412343 & 3.213006 & -0.411 & -0.000\end{array}$

$\mathrm{Ni}_{3}\left[\left(\mathrm{HSO}_{2}\right)_{2}-\mathrm{BAP}\right)_{4}\left(\mathrm{D}_{4}\right.$ symmetry $)$ Quintet 79 atoms

$\mathrm{E}=-3223.507439$ Hartrees $\mathrm{S}^{2}=6.0124$

$\begin{array}{rrrrrr}\mathrm{Ni} & 0.000000 & 0.000000 & 2.444289 & -0.088 & 1.663 \\ \mathrm{Ni} & 0.000000 & 0.000000 & 0.000000 & 0.135 & 0.099 \\ \mathrm{Ni} & 0.000000 & 0.000000 & -2.444289 & -0.088 & 1.663 \\ \mathrm{~N} & -0.920995 & 1.848415 & 2.150657 & -0.554 & 0.059 \\ \mathrm{~N} & 0.000000 & 1.964181 & 0.000000 & -0.367 & 0.005 \\ \mathrm{~N} & 0.920995 & 1.848415 & -2.150657 & -0.554 & 0.059 \\ \mathrm{C} & 0.497247 & 2.643476 & -1.091411 & 0.593 & -0.003 \\ \mathrm{C} & 0.525509 & 4.059555 & -1.093518 & -0.339 & 0.006 \\ \mathrm{C} & 0.000000 & 4.749299 & 0.000000 & -0.149 & -0.002 \\ \mathrm{C} & -0.525509 & 4.059555 & 1.093518 & -0.339 & 0.006 \\ \mathrm{C} & -0.497247 & 2.643476 & 1.091411 & 0.593 & -0.003 \\ \mathrm{H} & 0.963145 & 4.587994 & -1.927798 & 0.252 & -0.000 \\ \mathrm{H} & 0.000000 & 5.837284 & 0.000000 & 0.209 & 0.001 \\ \mathrm{H} & -0.963145 & 4.587994 & 1.927798 & 0.252 & -0.000 \\ \mathrm{~N} & -1.848415 & -0.920995 & 2.150657 & -0.554 & 0.059 \\ \mathrm{~N} & -1.964181 & 0.000000 & 0.000000 & -0.367 & 0.005 \\ \mathrm{~N} & -1.848415 & 0.920995 & -2.150657 & -0.554 & 0.059 \\ \mathrm{C} & -2.643476 & 0.497247 & -1.091411 & 0.593 & -0.003 \\ \mathrm{C} & -4.059555 & 0.525509 & -1.093518 & -0.339 & 0.006\end{array}$




\begin{tabular}{|c|c|c|c|c|c|}
\hline $\mathrm{C}$ & -4.749299 & 0.000000 & 0.000000 & -0.149 & -0.002 \\
\hline $\mathrm{C}$ & -4.059555 & -0.525509 & 1.093518 & -0.339 & 0.006 \\
\hline $\mathrm{C}$ & -2.643476 & -0.497247 & 1.091411 & 0.593 & -0.003 \\
\hline $\mathrm{H}$ & -4.587994 & 0.963145 & -1.927798 & 0.252 & -0.000 \\
\hline $\mathrm{H}$ & -5.837284 & 0.000000 & 0.000000 & 0.209 & 0.001 \\
\hline $\mathrm{H}$ & -4.587994 & -0.963145 & 1.927798 & 0.252 & -0.000 \\
\hline $\mathrm{N}$ & 0.920995 & -1.848415 & 2.150657 & -0.554 & 0.059 \\
\hline $\mathrm{N}$ & 0.000000 & -1.964181 & 0.000000 & -0.367 & 0.005 \\
\hline $\mathrm{N}$ & -0.920995 & -1.848415 & -2.150657 & -0.554 & 0.059 \\
\hline $\mathrm{C}$ & -0.497247 & -2.643476 & -1.091411 & 0.593 & -0.003 \\
\hline $\mathrm{C}$ & -0.525509 & -4.059555 & -1.093518 & -0.339 & 0.006 \\
\hline $\mathrm{C}$ & 0.000000 & -4.749299 & 0.000000 & -0.149 & -0.002 \\
\hline $\mathrm{C}$ & 0.525509 & -4.059555 & 1.093518 & -0.339 & 0.006 \\
\hline $\mathrm{C}$ & 0.497247 & -2.643476 & 1.091411 & 0.593 & -0.003 \\
\hline $\mathrm{H}$ & -0.963145 & -4.587994 & -1.927798 & 0.252 & -0.000 \\
\hline $\mathrm{H}$ & 0.000000 & -5.837284 & 0.000000 & 0.209 & 0.001 \\
\hline $\mathrm{H}$ & 0.963145 & -4.587994 & 1.927798 & 0.252 & -0.000 \\
\hline $\mathrm{N}$ & 1.848415 & 0.920995 & 2.150657 & -0.554 & 0.059 \\
\hline $\mathrm{N}$ & 1.964181 & 0.000000 & 0.000000 & -0.367 & 0.005 \\
\hline $\mathrm{N}$ & 1.848415 & -0.920995 & -2.150657 & -0.554 & 0.059 \\
\hline $\mathrm{C}$ & 2.643476 & -0.497247 & -1.091411 & 0.593 & -0.003 \\
\hline $\mathrm{C}$ & 4.059555 & -0.525509 & -1.093518 & -0.339 & 0.006 \\
\hline $\mathrm{C}$ & 4.749299 & 0.000000 & 0.000000 & -0.149 & -0.002 \\
\hline $\mathrm{C}$ & 4.059555 & 0.525509 & 1.093518 & -0.339 & 0.006 \\
\hline $\mathrm{C}$ & 2.643476 & 0.497247 & 1.091411 & 0.593 & -0.003 \\
\hline $\mathrm{H}$ & 4.587994 & -0.963145 & -1.927798 & 0.252 & -0.000 \\
\hline $\mathrm{H}$ & 5.837284 & 0.000000 & 0.000000 & 0.209 & 0.001 \\
\hline $\mathrm{H}$ & 4.587994 & 0.963145 & 1.927798 & 0.252 & -0.000 \\
\hline $\mathrm{S}$ & 1.603018 & 2.485508 & -3.467180 & 0.475 & 0.000 \\
\hline $\mathrm{S}$ & -1.603018 & -2.485508 & -3.467180 & 0.475 & 0.000 \\
\hline $\mathrm{S}$ & -1.603018 & 2.485508 & 3.467180 & 0.475 & 0.000 \\
\hline $\mathrm{S}$ & 1.603018 & -2.485508 & 3.467180 & 0.475 & 0.000 \\
\hline $\mathrm{H}$ & 0.583508 & 3.121988 & -4.106335 & 0.292 & -0.000 \\
\hline $\mathrm{H}$ & -0.583508 & -3.121988 & -4.106335 & 0.292 & -0.000 \\
\hline $\mathrm{H}$ & 0.583508 & -3.121988 & 4.106335 & 0.292 & -0.000 \\
\hline $\mathrm{H}$ & -0.583508 & 3.121988 & 4.106335 & 0.292 & -0.000 \\
\hline $\mathrm{O}$ & 1.962921 & 1.347799 & -4.332186 & -0.394 & 0.003 \\
\hline $\mathrm{O}$ & -1.962921 & -1.347799 & -4.332186 & -0.394 & 0.003 \\
\hline $\mathrm{O}$ & 1.962921 & -1.347799 & 4.332186 & -0.394 & 0.003 \\
\hline $\mathrm{O}$ & -1.962921 & 1.347799 & 4.332186 & -0.394 & 0.003 \\
\hline $\mathrm{O}$ & 2.619930 & 3.511848 & -3.188673 & -0.415 & 0.005 \\
\hline $\mathrm{O}$ & -2.619930 & -3.511848 & -3.188673 & -0.415 & 0.005 \\
\hline $\mathrm{O}$ & -2.619930 & 3.511848 & 3.188673 & -0.415 & 0.005 \\
\hline $\mathrm{O}$ & 2.619930 & -3.511848 & 3.188673 & -0.415 & 0.005 \\
\hline $\mathrm{S}$ & 2.485508 & -1.603018 & -3.467180 & 0.475 & 0.000 \\
\hline $\mathrm{S}$ & -2.485508 & 1.603018 & -3.467180 & 0.475 & 0.000 \\
\hline $\mathrm{S}$ & 2.485508 & 1.603018 & 3.467180 & 0.475 & 0.000 \\
\hline S & -2.485508 & -1.603018 & 3.467180 & 0.475 & 0.000 \\
\hline $\mathrm{H}$ & 3.121988 & -0.583508 & -4.106335 & 0.292 & -0.000 \\
\hline $\mathrm{H}$ & -3.121988 & 0.583508 & -4.106335 & 0.292 & -0.000 \\
\hline
\end{tabular}




$\begin{array}{lrrrrr}\mathrm{H} & -3.121988 & -0.583508 & 4.106335 & 0.292 & -0.000 \\ \mathrm{H} & 3.121988 & 0.583508 & 4.106335 & 0.292 & -0.000 \\ \mathrm{O} & 1.347799 & -1.962921 & -4.332186 & -0.394 & 0.003 \\ \mathrm{O} & -1.347799 & 1.962921 & -4.332186 & -0.394 & 0.003 \\ \mathrm{O} & -1.347799 & -1.962921 & 4.332186 & -0.394 & 0.003 \\ \mathrm{O} & 1.347799 & 1.962921 & 4.332186 & -0.394 & 0.003 \\ \mathrm{O} & 3.511848 & -2.619930 & -3.188673 & -0.415 & 0.005 \\ \mathrm{O} & -3.511848 & 2.619930 & -3.188673 & -0.415 & 0.005 \\ \mathrm{O} & 3.511848 & 2.619930 & 3.188673 & -0.415 & 0.005 \\ \mathrm{O} & -3.511848 & -2.619930 & 3.188673 & -0.415 & 0.005\end{array}$

$\mathrm{Ni}_{3}\left[\left(\mathrm{HSO}_{2}\right)_{2}-\mathrm{BAP}\right)_{4}\left(\mathrm{D}_{4}\right.$ symmetry $)$ Antiferromagnetic singlet 79 atoms $E=-3223.50921$ Hartrees $\mathrm{S}^{2}=1.9845$

\begin{tabular}{|c|c|c|c|}
\hline $\mathrm{Ni}$ & 0.000000 & 0.000000 & 2.429917 \\
\hline $\mathrm{Ni}$ & 0.000000 & 0.000000 & 0.000000 \\
\hline $\mathrm{Ni}$ & 0.000000 & 0.000000 & -2.429917 \\
\hline $\mathrm{N}$ & -0.926149 & 1.846096 & 2.146830 \\
\hline $\mathrm{N}$ & 0.000000 & 1.963547 & 0.000000 \\
\hline $\mathrm{N}$ & 0.926149 & 1.846096 & -2.146830 \\
\hline $\mathrm{C}$ & 0.500270 & 2.642264 & -1.090222 \\
\hline $\mathrm{C}$ & 0.528161 & 4.058379 & -1.092307 \\
\hline $\mathrm{C}$ & 0.000000 & 4.748037 & 0.000000 \\
\hline $\mathrm{C}$ & -0.528161 & 4.058379 & 1.092307 \\
\hline $\mathrm{C}$ & -0.500270 & 2.642264 & 1.090222 \\
\hline $\mathrm{H}$ & 0.967071 & 4.586630 & -1.926045 \\
\hline $\mathrm{H}$ & 0.000000 & 5.836015 & 0.000000 \\
\hline $\mathrm{H}$ & -0.967071 & 4.586630 & 1.926045 \\
\hline $\mathrm{N}$ & -1.846096 & -0.926149 & 2.146830 \\
\hline $\mathrm{N}$ & -1.963547 & 0.000000 & 0.000000 \\
\hline $\mathrm{N}$ & -1.846096 & 0.926149 & -2.146830 \\
\hline $\mathrm{C}$ & -2.642264 & 0.500270 & -1.090222 \\
\hline $\mathrm{C}$ & -4.058379 & 0.528161 & -1.092307 \\
\hline $\mathrm{C}$ & -4.748037 & 0.000000 & 0.000000 \\
\hline $\mathrm{C}$ & -4.058379 & -0.528161 & 1.092307 \\
\hline $\mathrm{C}$ & -2.642264 & -0.500270 & 1.090222 \\
\hline $\mathrm{H}$ & -4.586630 & 0.967071 & -1.926045 \\
\hline $\mathrm{H}$ & -5.836015 & 0.000000 & 0.000000 \\
\hline $\mathrm{H}$ & -4.586630 & -0.967071 & 1.926045 \\
\hline $\mathrm{N}$ & 0.926149 & -1.846096 & 2.146830 \\
\hline $\mathrm{N}$ & 0.000000 & -1.963547 & 0.000000 \\
\hline $\mathrm{N}$ & -0.926149 & -1.846096 & -2.146830 \\
\hline $\mathrm{C}$ & -0.500270 & -2.642264 & -1.090222 \\
\hline $\mathrm{C}$ & -0.528161 & -4.058379 & -1.092307 \\
\hline $\mathrm{C}$ & 0.000000 & -4.748037 & 0.000000 \\
\hline $\mathrm{C}$ & 0.528161 & -4.058379 & 1.092307 \\
\hline
\end{tabular}




\begin{tabular}{|c|c|c|c|}
\hline $\mathrm{C}$ & 0.500270 & -2.642264 & 1.090222 \\
\hline $\mathrm{H}$ & -0.967071 & -4.586630 & -1.926045 \\
\hline $\mathrm{H}$ & 0.000000 & -5.836015 & 0.000000 \\
\hline $\mathrm{H}$ & 0.967071 & -4.586630 & 1.926045 \\
\hline $\mathrm{N}$ & 1.846096 & 0.926149 & 2.146830 \\
\hline $\mathrm{N}$ & 1.963547 & 0.000000 & 0.000000 \\
\hline $\mathrm{N}$ & 1.846096 & -0.926149 & -2.146830 \\
\hline $\mathrm{C}$ & 2.642264 & -0.500270 & -1.090222 \\
\hline $\mathrm{C}$ & 4.058379 & -0.528161 & -1.092307 \\
\hline $\mathrm{C}$ & 4.748037 & 0.000000 & 0.000000 \\
\hline $\mathrm{C}$ & 4.058379 & 0.528161 & 1.092307 \\
\hline $\mathrm{C}$ & 2.642264 & 0.500270 & 1.090222 \\
\hline $\mathrm{H}$ & 4.586630 & -0.967071 & -1.926045 \\
\hline $\mathrm{H}$ & 5.836015 & 0.000000 & 0.000000 \\
\hline $\mathrm{H}$ & 4.586630 & 0.967071 & 1.926045 \\
\hline $\mathrm{S}$ & 1.607884 & 2.481906 & -3.464202 \\
\hline $\mathrm{S}$ & -1.607884 & -2.481906 & -3.464202 \\
\hline $\mathrm{S}$ & -1.607884 & 2.481906 & 3.464202 \\
\hline $\mathrm{S}$ & 1.607884 & -2.481906 & 3.464202 \\
\hline $\mathrm{H}$ & 0.588093 & 3.116699 & -4.104376 \\
\hline $\mathrm{H}$ & -0.588093 & -3.116699 & -4.104376 \\
\hline $\mathrm{H}$ & 0.588093 & -3.116699 & 4.104376 \\
\hline $\mathrm{H}$ & -0.588093 & 3.116699 & 4.104376 \\
\hline $\mathrm{O}$ & 1.969741 & 1.343997 & -4.327950 \\
\hline $\mathrm{O}$ & -1.969741 & -1.343997 & -4.327950 \\
\hline $\mathrm{O}$ & 1.969741 & -1.343997 & 4.327950 \\
\hline $\mathrm{O}$ & -1.969741 & 1.343997 & 4.327950 \\
\hline $\mathrm{O}$ & 2.623550 & 3.509559 & -3.185798 \\
\hline $\mathrm{O}$ & -2.623550 & -3.509559 & -3.185798 \\
\hline $\mathrm{O}$ & -2.623550 & 3.509559 & 3.185798 \\
\hline $\mathrm{O}$ & 2.623550 & -3.509559 & 3.185798 \\
\hline S & 2.481906 & -1.607884 & -3.464202 \\
\hline S & -2.481906 & 1.607884 & -3.464202 \\
\hline S & 2.481906 & 1.607884 & 3.464202 \\
\hline S & -2.481906 & -1.607884 & 3.464202 \\
\hline $\mathrm{H}$ & 3.116699 & -0.588093 & -4.104376 \\
\hline $\mathrm{H}$ & -3.116699 & 0.588093 & -4.104376 \\
\hline $\mathrm{H}$ & -3.116699 & -0.588093 & 4.104376 \\
\hline $\mathrm{H}$ & 3.116699 & 0.588093 & 4.104376 \\
\hline $\mathrm{O}$ & 1.343997 & -1.969741 & -4.327950 \\
\hline $\mathrm{O}$ & -1.343997 & 1.969741 & -4.327950 \\
\hline $\mathrm{O}$ & -1.343997 & -1.969741 & 4.327950 \\
\hline $\mathrm{O}$ & 1.343997 & 1.969741 & 4.327950 \\
\hline $\mathrm{O}$ & 3.509559 & -2.623550 & -3.185798 \\
\hline $\mathrm{O}$ & -3.509559 & 2.623550 & -3.185798 \\
\hline $\mathrm{O}$ & 3.509559 & 2.623550 & 3.185798 \\
\hline $\mathrm{O}$ & -3.509559 & -2.623550 & 3.185798 \\
\hline
\end{tabular}

